\title{
Efficient Routing Protocols and Coverage Issues in Wsn
}

\author{
Umashankar Pandey, Ashok Bhansali
}

\begin{abstract}
Wireless sensor network is the most favorable topic in the current era, as the technology is growing tremendously the size of the devices are decreasing gradually and hence the sensors are getting smaller and inexpensive. This makes the deployment of wireless sensor network less complicated, but not only size of the sensor is the problem other different issues are also there like coverage and connectivity issues, without proper coverage of the monitoring area and isolated sensor nodes will never form a proper network. There are different routing protocols which are prominent for the evolution of the wireless sensor network. In this survey we are going to discuss these coverage and connectivity issue and different routing protocols, so that it can help in future research work.
\end{abstract}

Keywords: Wireless sensor network, Sensor networks, Coverage \& Connectivity, Routing protocol, QoS.

\section{INTRODUCTION}

A group of sensor node which is spatially distributed over a wide area for monitoring the area is called wireless sensor network. The node and sink are the most important part of the wireless sensor network. Node collects the data from the monitoring area and sends it to sink or base station, node is small and low-processing device. Whereas sink is the important entity which can be define as a base station or as a gateway, data collector or can also be describe as access point for human interface. To establish a wireless sensor network with superlative quality of service (QoS) and considerable energy conservation we need to focus on the coverage and connectivity issues. Node scheduling problem is the basic problem in which we have to minimize the number of sensor nodes which are required to actively participate for coverage of the monitoring area, by solving the node scheduling problem be can obtain a greater coverage with less energy consumption. It is important to maintain connectivity along with coverage in sensor network [2]. Each sensor node has its own sensing range and communication range which is used to sense and communicate with other sensor node for data transfer from sensor node to the base station.

By achieving proper connectivity and coverage we get better QoS with less energy consumption.

Revised Manuscript Received on February 05, 2020.

* Correspondence Author

Umashankar Pandey*, CSE, O.P.Jindal University, Raigarh, India. Email: umashankar.pandey@opju.ac.in

Ashok Bhansali, CSE, O.P.Jindal University, Raigarh, India. Email: ashok.bhansali@opju.ac.in

(c) The Authors. Published by Blue Eyes Intelligence Engineering and Sciences Publication (BEIESP). This is an open access article under the CC BY-NC-ND license (http://creativecommons.org/licenses/by-nc-nd/4.0/)
Another predominant area is the routing protocols for wireless sensor network. To achieve greater quality of service we need to achieve proper communication between nodes for that a mechanism needed in which nodes can request and advertise data and publicize that data to interested nodes. In this paper, we analyze coverage and connectivity issue related problem solving strategies and mechanism Along with the coverage and connectivity issue we define the routing protocol of WSN.

\section{COVERAGE \& CONNECTIVITY ISSUES}

The basal issue in wireless sensor network is the coverage and connectivity issues. To develop a wireless sensor network with high quality of service (QoS) and energy conservation we have to focus on how well we can resolve these issues. In coverage problem the center attention is how adeptly we can cover the monitoring area with small number of sensor nodes involvement; node scheduling problem is the basic problem to focus to minimize the coverage issues this results in huge energy conservation. Connectivity can be defined as how swiftly a sensor node can rout data to sink or base station through other sensor node or directly. If there is no possible, route is available then it becomes difficult to rout data which hinder the quality of service and consume vast amount of energy. So it is very important to maintain coverage along with connectivity in wireless sensor network. Below is the detail through which the sensing range can be calculated.

\section{A. Condition for Intersection}

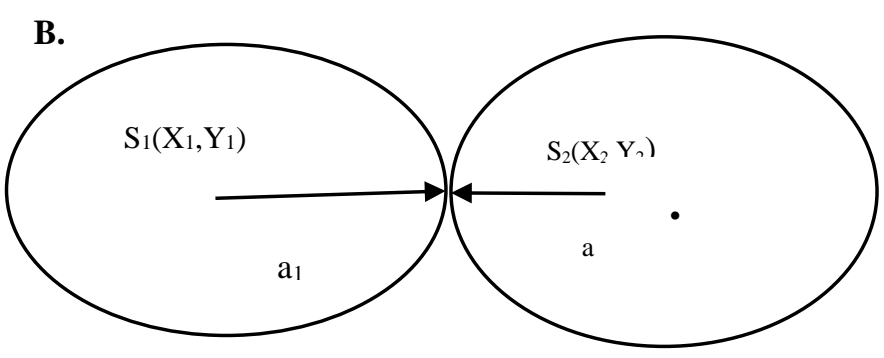

Figure1. Condition for intersection of two sensors In fig1 there are two sensor nodes S1 and S2, let A be the area covered by S1 and B be the area covered by S2. The two sensor nodes intersect with each other when sum of radii is less then and equal to distance between centers.

Distance between center

$$
=\sqrt{\left(\left(X_{2}-X_{1}\right)^{2}+\left(Y_{2}-Y_{1}\right)^{2}\right)}
$$

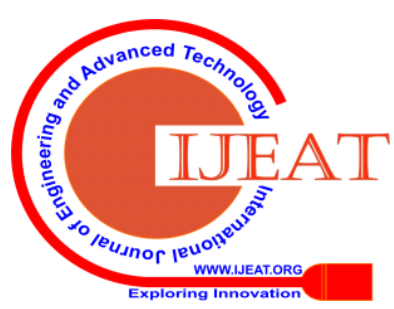


Hence,

$$
\begin{aligned}
& \mathrm{a}_{1}+\mathrm{a}_{2} \leq \sqrt{\left(\left(X_{2}-X_{1}\right)^{2}+\left(Y_{2}-Y_{1}\right)^{2}\right)} \\
& \mathrm{a}_{1}+\mathrm{a}_{2} \leq \mathrm{D}(\mathrm{S} 1, \mathrm{~S} 2)
\end{aligned}
$$

- When sensor S1 and S2 sensing range separate with each other then it can be calculated as-

$$
\mathrm{D}(\mathrm{s} 1, \mathrm{~s} 2)>=\mathrm{a} 1+\mathrm{a} 2 \text { means } \mathrm{A} \cap \mathrm{B}=\varnothing
$$

- When sensor S1and S2 sensing range intersect with each other creating any area can be calculated as

$$
\mathrm{a} 1-\mathrm{a} 2<\mathrm{D}\left(\mathrm{s}_{1}, \mathrm{~s}_{2}\right)<\mathrm{a} 1+\mathrm{a} 2 \text { Means } \mathrm{A} \cap \mathrm{B} \neq \varnothing
$$

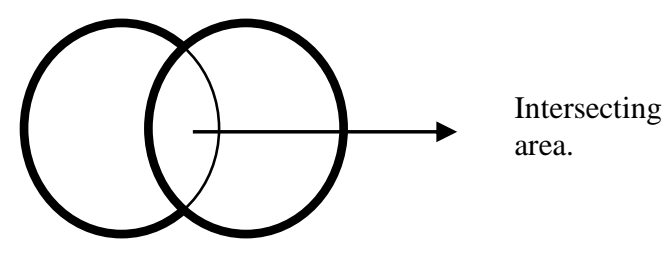

Figure2. Two Intersecting Sensors

\section{C.Sleep Scheduling Mechanism}

Sensors are deployed in the monitoring area from long run; it become arduous to change the sensor if it gets discharged hence its energy conservation is of preeminent concern. Sleep scheduling mechanism is an efficient mechanism for energy conservation in which the sleep interval of irrelevant nodes is scheduled and only the nodes which are necessary for observation are active. The active node will cover the physical area and maintain connectivity for better QoS. This mechanism will save huge amount of energy wastage [7]. In this case the difficulty arises is the different set of modes and application of nodes. Some nodes are on sensing phase some are on sensing unit on-duty, while some are on transaction phase and some are on off-duty.

\section{Disjoint Dominating Sets}

To maintain the energy conservation and increasing network lifetime is a challenging issue. Disjoint dominating sets is the mechanism in which all the deployed sensor nodes in the monitoring area are divided in disjoint sets, it is preferred to make as many disjoint set as possible. Each disjoint set can able to monitor the whole physical area, when one disjoint set is activated then other nodes or sets are in sleep mode, it will increase the network lifetime as well as conserve energy [20].

\section{Self-scheduling strategy:}

Wireless sensor network needs some strong strategy and algorithm to achieve more network lifetime and energy conservation. Self-scheduling strategy is developed in which the main concept is to minimize the number of active nodes, such that the full coverage can be achieved with minimal number of active node. To achieve maximum coverage with minimum number of active node different algorithm are been proposed like off-duty eligibility rule in which eligible nodes will turn off their processing, Layered diffusion based coverage control (LDCC), Connected K-Neighbourhood (CKN).

\section{D.Coverage Deployment Strategy}

By maintaining the low cost deployment, we can be maximizing the coverage and optimize the resources in the network or sensing field. When the network area is unknown and unpredictable then it is very challenging to maximize coverage. Coverage deployment strategies can be divided into static coverage and dynamic coverage.

\section{1) Static Coverage}

In static coverage the nodes in the wireless sensor network are stationery, in most of the cases the deployment is predefined. In static coverage the monitoring area can be fully covered, each target is sensed by at least one sensor. Static coverage area can be defined in three types:

\section{a) Efficient Coverage Area}

To achieve optimum coverage in a predefined sensing area the deployment of sensor nodes is according to the shape of the network area. In the monitoring space a particular area can be covered by multiple nodes hence causes redundancy, to remove this problem efficient coverage area method is used in which the overlapping area is subtracted from the total sensing area of a node. Overlapping area is the region which is covered by multiple nodes [4].

\section{b) k-coverage}

For an adequate coverage in an harsh area where it is difficult to reach the sensor node for any change or replacement k-coverage methodology is used in which the deployed sensor nodes are divided in number of cluster or groups, each cluster sense a particular region of the network and it is checked that each region is been sense by at least two sensor node, here ' $k$ ' defines the minimum number of sensor node to sense each point in the physical area.

\section{c) Path Coverage}

In path coverage the node are placed to secure a particular path and report possible efforts made by intruders to cross it. There are two types of deployment are made: manual deployment, the desired level of path coverage is achieved when proper placement is done for coverage, second is random deployment, when it is not possible to do manual deployment in which nodes are deployed by dropping sensors from an aircraft, is used [5].

\section{2) Dynamic Coverage}

In dynamic coverage all the nodes can move they are mobile in nature. As the node has the ability to move, this makes the dynamic coverage more difficult. The placement of nodes become difficult in environment which is unspecified or mysterious hence the deployment is made random [6]. There are different dynamic coverage strategies as follows:

\section{a) Based on virtual force}

In dynamic coverage when the node placement is done randomly after that to maximize the coverage virtual force algorithm (VFA) is used for deployment strategy.

The idea of virtual force algorithm is referenced from the basic physics force model, like the gravitation and Coulomb force between molecules, elastic force etc [18].

$$
\text { Coulomb force: } F=k \frac{q 1 q^{2}}{r^{2}} \text {, Gravitation: } F=G \frac{m M}{r^{2}}
$$

Where: q1, q2 are power of electric charge; and $\mathrm{r}$ is distance between them; $\mathrm{K}$ is a constant. ' $\mathrm{M}$ ' and $\mathrm{m}$ are quality, $\mathrm{G}$ is a constant. In [21]

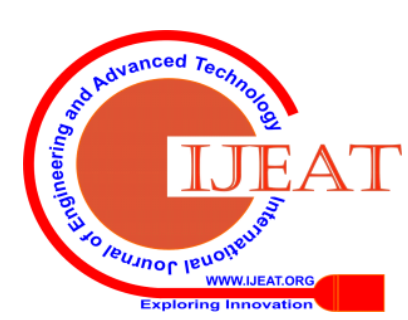


assume that repulsive force is applied by obstacles and attractive force is applied by the area preferential coverage on a sensor. Let $\overrightarrow{F_{m A}}$ be the attractive force on $X_{m}$ and $\overrightarrow{F_{m R}}$ be the repulsive force on $X_{m}$. The total force $\overrightarrow{F_{m}}$ on $X_{m}$ can be convey as-

$$
\overrightarrow{F_{m}}=\sum_{n=1, n \neq m}^{k} \overrightarrow{F_{m n}}+\overrightarrow{F_{m R}}+\overrightarrow{F_{m A}}
$$

Then The force $\overrightarrow{F_{m}}$ between $\mathrm{X}_{\mathrm{m}}$ and $\mathrm{X}_{\mathrm{n}}$ in polar Coordination can be conveying as

$$
\begin{aligned}
& \left.\overrightarrow{F_{m n}}=\left(W_{A}\left(d_{m n}-d_{t h}\right), \alpha_{m n}\right)\right) \ldots . . . \text { if }\left(d_{m n}>d_{t h}\right) \\
& \overrightarrow{F_{m n}}=0 \text {..........................if }\left(d_{m n}=d_{t h}\right) \\
& \left.\overrightarrow{F_{m n}}=\left(W_{A}\left(1 / d_{m n}\right), \alpha_{m n}+\pi\right)\right) \ldots \ldots . . . i f\left(d_{m n}>d_{t h}\right)
\end{aligned}
$$

Here $d_{m n}$ is Euclidean between sensor $X_{m}$ and $X_{n}, d_{t h}$ is threshold on the distance between $X_{m}$ and $X_{n}, \alpha_{m n}$ is orientation of a line segment $\mathrm{X}_{\mathrm{m}}$ and $\mathrm{X}_{\mathrm{n}}$ and $\mathrm{W}_{\mathrm{A}}$ is the attractive force measurement.

\section{b) Graph-based}

Voronoi diagram based approach is a geometrical approach to achieve coverage and connectivity. In this approach the node as placed in the form of a polygon. Fig 3 shows the Voronoi diagram which is based on the concept of electromagnetic particles, when electromagnetic particles come close to each other then they exert a repulsive force which pushes them apart. Similar methodology is applied on sensor node in which when the sensor node come closer with the average distance then they exert a force on them which pushes them apart. [17].

\section{E. Repair policies of coverage hole}

Nodes in wireless sensor network are deployed for long term use, there may be a case occurs when a node stop working or get damaged which create a coverage hole in the network and hinders connectivity. Very few algorithms are developed for coverage hole recovery, the main idea in which the algorithm of recovery works is to regain the connectivity with the spare nodes [4].

\section{F. Adjustable coverage radius}

We can enhance the coverage and connectivity by adjusting the coverage radius of sensor node, every sensor node has its sensing range which is adjustable. By adjusting the node radius, we can remove the overlapping area and can save energy with maintaining connectivity. There are different algorithm and methodology are developed which use adjustable coverage radius for maintain coverage and connectivity for QoS. Target coverage problem in which m-targets with known location and n-sensor node to cover all those targets observe continuously. Radius adaptive mechanism is used which is a mathematical model in which linier programming; heuristic model and greedy technique is used to solve the Adjustable range set cover (AR-SC) problem.

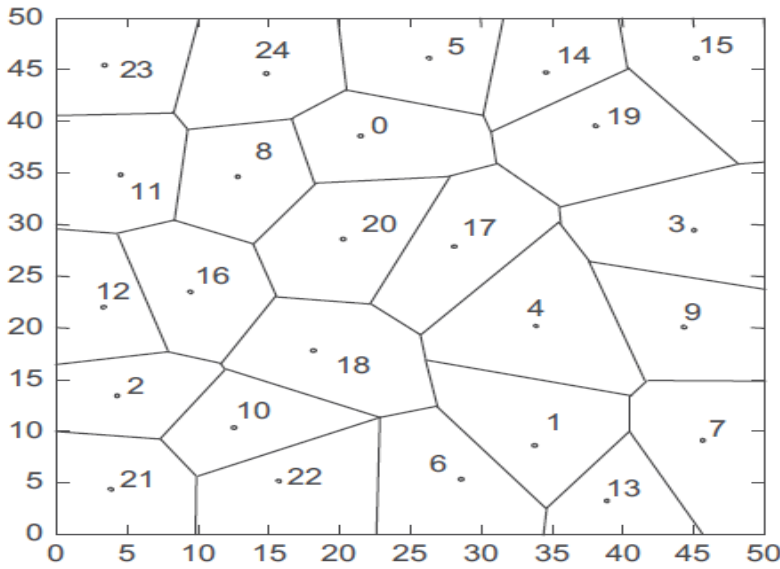

Figure 3. Voronoi Diagram [17]

\section{WSN ROUTING PROTOCOLS}

Data transferring is the supreme function of the wireless sensor network so to transfer data in WSN certain set of rules or protocol are established. There are many issues which led to the formation of these protocols like system architecture and design issue, coverage and connectivity issues, as different WSN has different architectural design so we can't follow a particular set of rule to transfer data in every network system. Some sensor nodes architecture is stationery whereas some have mobile sensor node and sink, it is challenging to route messages in mobile network because steadiness becomes a chief optimization factor with energy and bandwidth. The deployment is either deterministic or self-organizing. Almost all the WSN routing protocol can be categorized as data-centric, hierarchical and location based whereas there are few other left which are distinct from above are based on network flow or quality of service (QoS) awareness fig2 shows the different classification of routing protocols. Each categories of routing protocol are based on specific methodology. Like in hierarchical protocol the nodes which are deployed in the monitoring region are for into number of clusters with one cluster head each. It will reduce the transmission of redundant data and location based protocol have the location information of every node in the region which helps in forwarding the data to desired region easily. Lastly the network flow and QoS-aware protocol that aspire for meeting some QoS requirements along with the routing function.

\section{A.Data-centric protocols}

In WSN the sensor nodes are deployed in verities of way depending on the application, in some application few nodes can do the work while in other voluminous number of nodes needed. Earlier the nodes can communicate with each other through global identifiers but the problem arrives when there is immense number of node, then it became difficult to provide each and every node with global identifier, it consumes huge amount of energy to transfer every data to every node with or without demand, and therefore an algorithm needed in which some group of node is selected and data aggregation can be done during transmission [10] Data centric routing work on attribute based routing.

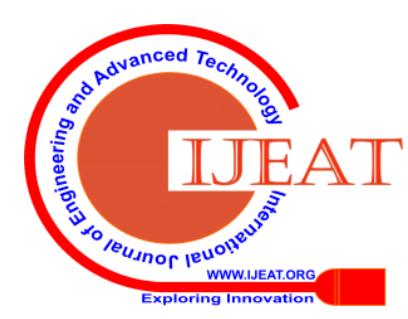




\section{Efficient Routing Protocols and Coverage Issues in Wsn}

\section{1) Flooding and gossiping}

In Flooding when a sensor node need to transmit data to a particular destination then it starts sending data to all of its neighbor nodes until the destination reached. Its only advantage is easy to implement. Whereas slightly enhanced version of flooding is gossiping in which when data transmission takes place then the node sends the data to randomly selected neighbor nodes and those selected nodes will forward another randomly selected neighbor nodes until the destination reached [9].

\section{2) Sensor protocols for information via negotiation} (SPIN):

SPIN is the first data centric routing protocol. The key feature of SPIN is that it uses advertisement mechanism. In SPIN meta-data is created and transmitted between nodes. Whenever a node receives a new data then it advertises it to its neighbor and interested neighbor. The process of sharing meta-data is complete in three steps they are the ADV message called advertise message or meta-data i.e. nodes do advertisement of its meta-data through ADV message. Secondly REQ message, whenever a node need a specific data then it sends a REQ massage and thirdly the DATA message used to transmit the actual data.

\section{3) Directed diffusion}

Routing data to each node in the network take extra energy and time to reduce this new mechanism developed called directed diffusion, it is different from SPIN in directed diffusion the data is broadcast by using naming scheme and attributes value pair. By using this pair value node can demand it at any time. Path through which data is transmitted can be repaired in directed diffusion; whenever path fails it reinitiates reinforcement by searching other path. It can also search the alternative path in advance which will reduce time and energy consumption when new path search is needed. Application which require continuous data delivery to the base station in which it is not possible to do directed diffusion because it works on query driven data transmission model [8].

\section{4) Energy-aware routing}

Energy-aware routing was proposed to increase the lifetime of the network. As the nodes are tiny and low processing power device therefore its energy decreases gradually in less time, hence it is very important to increase the lifetime. This routing protocol uses a sub-optimal path chosen through probability function; it is based on energy consumption of each path. The three phase in the protocol are:

a) Setup Phase

If the request is sent from node xi to node $\mathrm{xj}, \mathrm{xi}$ path cost is calculated as follows:

$$
C_{x j x i}=\operatorname{Cost}\left(x_{i}\right)+\operatorname{Metric}\left(x_{j}, x_{i}\right)
$$

b) Data communication phase.

c) Route maintenance phase.

\section{5) Rumor routing}

Rumor routing is different version of directed diffusion routing. It is an agent based path creation algorithm; it is mainly intended for applications where geographic routing is not feasible. The key idea is to route the queries to the nodes that have observed a particular event and do not flood it to entire network. In order to flood events through the network, the rumor routing algorithm employs long - lived packets, called agents.

\section{6) Gradient-based routing}

In gradient-based routing nodes can able to calculate the minimum number of hops between the nodes and sink. Each node can discover minimum hope count which is called as height, the difference between the hope counts of two nodes is called gradient. A packet is forwarded on a link with the minimum hope count, there are different data spreading techniques when two or next hope have same gradient then node choose any of them randomly this technique is called Stochastic scheme, in Energy-based scheme when a node energy goes below the threshold level then it increases its height and Stream-based scheme are used.

\section{7) CADR}

Constrained anisotropic diffusion routing protocol (CADR), it is general form of Directed Diffusion. To maximize information gain and minimizing latency and bandwidth it routes data in the network and query sensor node. It can be achieved by dynamically adjusting the route and selecting the sensors which are close to the event [5].

\section{8) COUGAR}

It views a network as a huge distributed database system. In the architecture of CAUGAR the sensor nodes select a head node between them which will transmit data and do aggregation. It uses declarative queries for processing like selection of sensor nodes etc.

\section{9) ACQUIRE}

It stands for active query forwarding in sensor network, it can do complex queries consist of sub-queries and view sensor node as a distributed database. The base station or the sink node forward data to the distributed nodes, by receiving the queries nodes partially respond to it and forward it to other nodes. When the query is fully resolved then it sent back to the sink node through shortest path.

\section{B. Hierarchical protocol}

Hierarchical protocol works on the scalability of the sensor network. If number of sensor node increases, then single-tier gateway gets overloaded.

The main aim of hierarchical routing is too adept with the additional load by efficiently maintain the energy consumption of sensor nodes. Hierarchical protocol maintains energy consumption through multi-hope communication, data aggregation and fusion.

\section{1) LEACH}

Low-energy adaptive clustering hierarchy (LEACH) is very popular routing algorithm. It forms a cluster of sensor nodes deployed in the monitoring area with a cluster head. Cluster head is responsible for data transmission to sink; it will reduce energy consumption because only the cluster head requires maximum energy for processing.

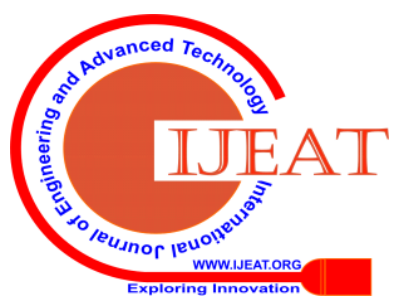




\section{2) PEGASIS and Hierarchical-PEGASIS}

Power efficient gathering in sensor information system (PEGASIS) it is different or improved version of LETCH because in PEGASIS it doesn't form cluster of node here it forms a chain of cluster which aggregate data from one another and only single node among them is responsible for transmission, this will much more energy consumption [11].

\section{3) TEEN and APTEEN}

Threshold sensitive Energy Efficient sensor network protocol (TEEN) it is designed to be responsive to sudden changes in the sensed attribute such as temperature. This protocol is important for time-critical application.

4) Energy-aware routing for cluster based sensor networks: Younis et al [12] proposed an algorithm based on three-tier architecture. Before network operation started the sensors are grouped into clusters. Cluster heads namely gateways, which are less energy constrained then sensors and are assume to know the location of sensor nodes. Gateway maintains the states of the sensors and set up multi-hop routes for collecting sensors data.

5) Self-organized protocol: The architecture of self-organized protocol supports the sensor node which are mobile and can also be stationery this sensor node forward data to destination and work as a router. The nodes which are connected to router are easily addressable [16]

\section{Location-Based Protocol:}

In location - based protocol the location information of sensor nodes are known, this information is very benevolent because the queries send to the particular node which is interested not to other this will save energy. This protocol works on both mobile as well as stationery sensor nodes [12].

\section{1) MECN \& SMECN}

MECN stands for Minimum energy communication network it uses low power GPS for maintaining minimum energy network for WSN. It can work for mobile network but is well suited for stationery network. SMECN stands for small minimum energy communication network is an augmentation of MECN.

\section{2) GAF}

Geographic adaptive fidelity its main idea is formation of virtual grid for the monitoring area in which the nodes are associated with a point in virtual grid through GPS-indicated location. GAF primarily works for mobile ad-hoc networks but can be applicable in WSN also.

\section{3) GEAR}

Geographic \& energy aware routing protocol is an idea to conserve energy by selecting nodes through geographical information and forward data to destination. It considers only few regions for data transmission and does not forward it to whole network.

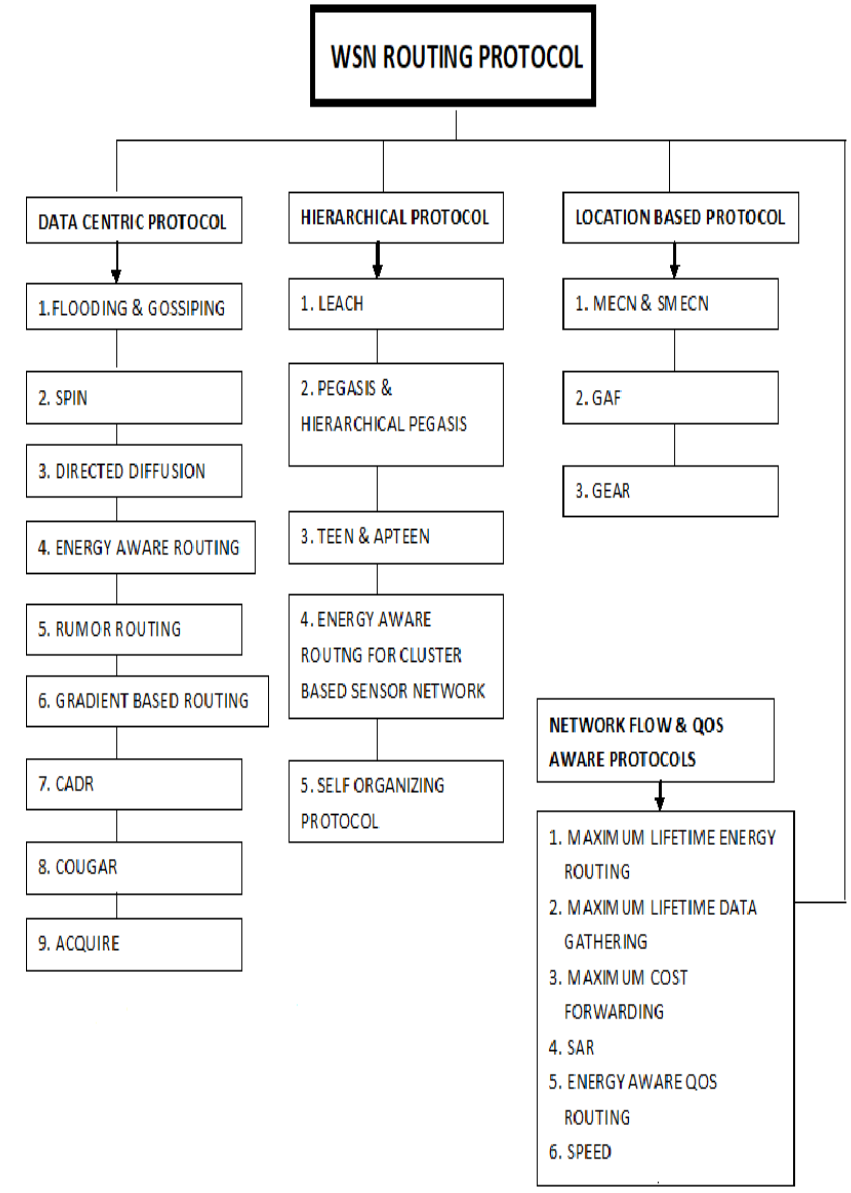

Figure 4. Classification of different routing protocol

\section{Network flow \& qos-aware protocols}

Most of all routing protocol can be considered under the above discussed routing protocols, but some are there which comes under network flow and QoS-aware protocol. In this protocol the routing problem or data flow problem is solved and end-to-end delay is maintained for better QoS.

1) Maximum lifetime energy routing

It calculates the function cost of transmission by calculation the total energy required for transmission and the energy left after transmission. This routing save the energy of the sensor network and maximize the lifetime of the network [13].

2) Maximum lifetime data gathering

It calculates the maximum time in which a node gets exhausted after a certain round of operation.

The lifetime of the network depends on the time duration in which the network schedule remains alive or active [14].

3) Minimum cost forwarding

It calculates the cost function of the network which consists of the effect of delay, energy consumption and throughput of node to the sink. It consists of two phases called the setup phase which set the cost value of each node. In second phase the source node transmit data to its neighbor by calculating the sufficient cost.

\section{4) SAR}

Sequential assignment routing (SAR) by taking each path in consideration and taking QoS it forms trees routed to the sink. 
By using this tree multiple paths from sink to node are formed. SAR also performs failure recovery by enforcing routing table.

\section{5) Energy-aware QoS routing protocol}

This protocol by imagining sensors generate a real-time traffic. It uses a function called link cost and calculate transmission energy, nodes reserved energy after transmission, delay etc. [15].

\section{6) SPEED}

The protocol use geographic location based forwarding for path discovery and maintains information about its neighbor nodes. Whenever the network gets congested the SPEED provide congestion avoidance consider certain speed for every packet in the network.

\section{RESULT AND DISCUSSION}

We performed experiments based on the mathematical model using MATLAB R2014a 8.3.0.532 on a computer system having configuration of Intel i7 processor, $3.4 \mathrm{GHz}$ CPU, 8 GB RAM and Microsoft Windows 10 as a platform. The result of average degree of coverage with different sensing ranges are showing in figure 5 , the coverage detection time with different nodes with different sensing ranges are showing by figure 6 (Degree of coverage for different nodes when Sensing Range equal to Communication Range, Communication Range twice of Sensing range and Communication Range greater than double of sensing range [21]).

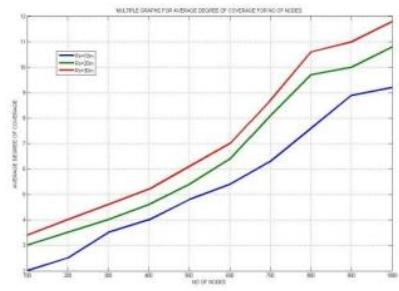

Figure 5. Average Degree of Coverage with different nodes [21] (50to 500) with Various Sensing Ranges(5M,10M,15M).

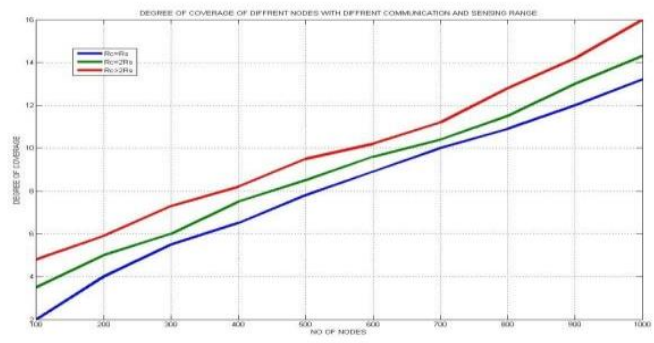

Figure 6. Degree of coverage for different Nodes when Communication Range equal to Sensing range,

Communication Range equal to Double of sensing range and Communication Range Greater than double of sensing range [21].

\section{CONCLUSION}

In this survey, we first gave an overview of wireless sensor network (WSN) and its properties, different problems related to coverage \& connectivity issues are discussed. We then covered two important topics of sensor network: Coverage \& Connectivity issue and routing protocols. We have taken into account different factors like QoS related problems, energy conservation, area coverage and delay problems have been discussed and tried to give proper solution to them. Also different research result of routing protocol and its uses, methodology is described; special category called network flow and QoS-aware protocol is also defined. This paper provides a general survey on recent developments in WSN and deliberates to help new researchers to enter in this domain.

\section{REFERENCES}

1. Jennifer Yick, Biswanath Mukherjee, Dipak Ghosal, "Wireless sensor network survey", ELSEVER 2008.

2. Hamid Barati, Ali Movaghar, Ali Barati, and Arash Azizi Mazreah, "A Review of Coverage and Routing for Wireless Sensor Networks", World Academy of Science, Engineering and Technology. International Journal of Electronics and Communication Engineering, 2008.

3. Wang X, Xing G, Zhang Y, Lu C, Pless R, Gill C.’'Integrated coverage and connectivity configuration in wireless sensor networks. In: Proceedings of the 1st international conference on embedded networked sensor systems", ser. SenSys'03. , New York, NY, USA: ACM; 2003.

4. Wang X, Zhang S. "Research on efficient coverage problem of node in wireless sensor networks. In: Electronic commerce and security", 2009. ISECS'09. Second international symposium, vol. 2; 2009.

5. Wu X, Cho J, D'auriol BJ, Lee S. ,'Mobility-assisted relocation for self-deployment" in wireless sensor networks 2007.

6. Liu B, Brass P, Dousse O, Nain P, Towsley D. "Mobility improves coverage of sensor networks. In: Proceedings of the 6th ACM international symposium on mobile ad hoc networking and computing", ser. MobiHoc'05. New York, NY, USA: ACM; 2005.

7. Yuan Z, Wang L, Shu L, Hara T, Qin Z. "A balanced energy consumption sleep scheduling algorithm in wireless sensor networks". In: International wireless communications and mobile computing conference (IWCMC-2011); 2011.

8. Cardei M, Thai M, Li Y, Wu W. "Energy-efficient target coverage in wireless sensor networks". In: INFOCOM 2005. 24th annual joint conference of the IEEE computer and communications societies. Proceedings IEEE, vol. 3; 2005a.

9. S. Hedetniemi, A. Liestman, "A survey of gossiping and broadcasting in communication networks", Networks 18 (4) (1988).

10.M. Chu, H. Haussecker, F. Zhao, "Scalable informationdriven sensor querying and routing for ad hoc heterogeneous sensor networks", The International Journal of High Performance Computing Applications 16 (3) (2002).

11.S. Lindsey, C.S. Raghavendra," PEGASIS: power efficient gathering in sensor information systems", in: Proceedings of the IEEE Aerospace Conference, Big Sky, Montana, March 2002.

12.M. Youssef, M. Younis, K. Arisha, A constrained shortestpath energy-aware routing algorithm for wireless sensor networks, in: Proceedings of the IEEE Wireless Commu-348 K. Akkaya, M. Younis / Ad Hoc Networks 3 (2005) 325-349 nication and Networks Conference (WCNC2002), Orlando, FL, March 2002.

13.J.-H. Chang, L. Tassiulas, "Maximum lifetime routing in wireless sensor networks", in: Proceedings of the Advanced Telecommunications and Information Distribution Research Program (ATIRP_2000), College Park, MD, March 2000.

14.K. Kalpakis, K. Dasgupta, P. Namjoshi, "Maximum lifetime data gathering and aggregation in wireless sensor networks", in: Proceedings of IEEE International Conference on Networking (NETWORKS 02), Atlanta, GA, August 2002.

15.K. Akkaya, M. Younis, "An energy-aware QoS routing protocol for wireless sensor networks”, (MWN 2003).

16.L. Subramanian, R.H. Katz, "An architecture for building self configurable systems", in: Proceedings of IEEE/ACM Workshop on Mobile Ad Hoc Networking and Computing, Boston, MA, August 2000.

17.Aurenhammer F,"Voronoi diagrams a survey of a fundamental geometric data structure". ACMComputingSurveys1991.

18.Liu YH, Chen ZY, Wu J, Xiong Z. "Proactive Wakeup and Sleep Scheduling Scheme for Localizing Mobile Sensors". Journal of Software. 2009, 1(20): 164-176

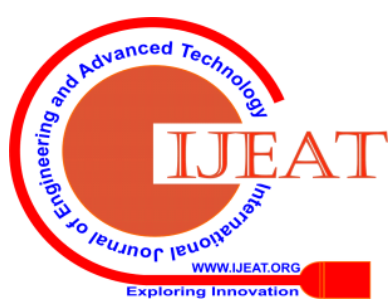


19.Kemal Akkaya, Mohamed Younis, "A survey on routing protocols for wireless sensor networks" ELSEVIER, 2005.

20.Chuan Zhu, Chunlin Zheng, Lei Shu, Guangjie Han,"A survey on coverage and connectivity issues in wireless sensor networks". ELSEVIER 2012.

21.Rasmi Ranjan Patra, Prashant Kumar Patra, "Analysis of k-Coverage in Wireless Sensor Network" IJACSA Vol. 2, No. 9, 2011.

22.Zou Y, Chakrabarty K. "Sensor deployment and target localization based on virtual forces". In: INFOCOM 2003. Twenty-second annual joint conference of the IEEE computer and communications. IEEE societies, vol. 2; 2003.

\section{AUTHORS PROFILE}

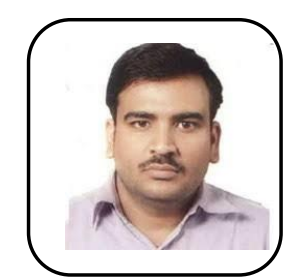

Umashankar Pandey Presently working as an assistant professor in O.P.Jindal University, Raigarh ,India.He is PursuingPh.D.From OPJU

Raigarh, India.His Ph.D. Work is in the field of Wireless Sensor Networks and main interest of research is on developing energy efficient routing and clustering algorithm with coverage and connectivity .

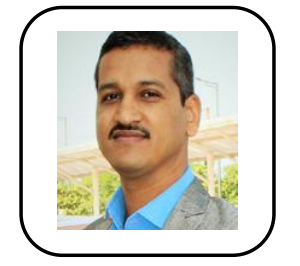

Prof. Ashok Bhansali received his B.Tech and M.Tech degree from NIT Jamshedpur and NIT Raipur respectively .He has completed his $\mathrm{PhD}$ degree in 2013 from Singhania University .Currently he is working as Professor in department of Computer Science and Engineering in OP Jindal University Raigarh, India. His current research interest includes wireless sensor networks, IoT, Machine leaning and Cloud Computing. 